\title{
Ambiances
}

anbiances Environnement sensible, architecture et espace urbain Comptes-rendus | 2014

\section{Carlotta Darò, Avant-gardes sonores en architecture}

Les Presses du Réel (collection Ohcetecho, arts sonores), Dijon, 2013

\section{Henry Torgue}

\section{OpenEdition}

1 Journals

Édition électronique

URL : http://journals.openedition.org/ambiances/424

DOI : $10.4000 / a m b i a n c e s .424$

ISSN : 2266-839X

Éditeur:

Direction Générale des Patrimoines - DAPA - MCC, UMR 1563 - Ambiances Architectures Urbanités (AAU)

\section{Référence électronique}

Henry Torgue, "Carlotta Darò, Avant-gardes sonores en architecture », Ambiances [En ligne], Comptes-

rendus, mis en ligne le 19 avril 2014, consulté le 22 septembre 2020. URL : http://

journals.openedition.org/ambiances/424 ; DOI : https://doi.org/10.4000/ambiances.424

Ce document a été généré automatiquement le 22 septembre 2020.

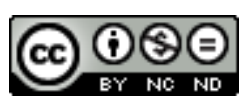

Ambiances is licensed under a Creative Commons Attribution-NonCommercial-NoDerivatives 4.0 International License. 


\title{
Carlotta Darò, Avant-gardes sonores en architecture
}

Les Presses du Réel (collection Ohcetecho, arts sonores), Dijon, 2013

\author{
Henry Torgue
}

\section{RÉFÉRENCE}

Carlotta Darò, Avant-gardes sonores en architecture, Les Presses du Réel (collection Ohcetecho, arts sonores), Dijon, 2013, 300 pages (dont 40 d'illustrations)

1 «L'architecture s'entend autant qu'elle se voit!» Cet aphorisme vient à l'esprit pour condenser l'ouvrage de Carlotta Darò qui interroge l'architecture du point de vue du sonore, à la fois comme dimension acoustique de l'espace et comme matière sensible du perçu.

2 D'emblée, rectifions trois fausses pistes sur le contenu du livre que pourrait suggérer le titre. Les avant-gardes dont il est question ici rassemblent davantage des expérimentations innovantes que des prises de position idéologiques ou politiques; les processus de création priment sur la dimension polémique. Deuxième rectification : la plupart des productions sonores présentées relèvent du domaine musical dans son acception élargie et ne concernent qu'exceptionnellement les pratiques habitantes; il ne s'agit pas d'architecture vécue. Enfin, troisième ajustement, l'interface de la rencontre entre les expériences sonores et les lieux est moins l'architecture en tant que processus d'édification, que l'espace dans ses multiples modulations, de la pièce à la ville.

3 Si l'on comprend donc le titre ainsi : Expérimentations sonores et localisation, les fausses attentes s'estompent et l'ouvrage se révèle être un passionnant catalogue, foisonnant de récits parfaitement documentés et alimentant une réflexion très riche sur les relations du sonore, dans ses expressions savantes, avec l'espace. Sans barrières stylistique ou esthétique, ce travail explore des recherches croisant l'acoustique, l'écriture musicale, les matières sonores, les dynamiques de diffusion, l'implantation 
phonique, les interactions entre formes audibles et formes visuelles, et jusqu'aux questions environnementales posées par l'écologie sonore.

4 Le $\mathrm{XX}^{\mathrm{e}}$ siècle $\mathrm{y}$ est très présent en tant que creuset particulièrement productif ayant exploré deux voies principales : celle du développement de l'enregistrement et des procédés de reproduction de masse, et celle de l'exploration de l'espace comme instrument-propagateur jouant avec les instruments-sources. Grâce à cette double évolution, technologique et scénographique, la musique dans ses divers courants, et plus largement les arts du sonore, ont produit non seulement des œuvres à écouter mais des configurations mentales débouchant sur de nouvelles formes, spatiales ou sociales. Le progrès des technologies électro-acoustiques et celui du contrôle environnemental ont engendré de profondes transformations dans la perception classique du son et de l'espace bâti.

5 Il est très agréable de retrouver ou de découvrir au fil des pages de Carlotta Darò les récits détaillés des pionniers qui contribuèrent à cette aventure. De Adolf Loos à Archigram en architecture, de Erik Satie à Robert Murray Schafer en musique, en passant par Nicolas Schöffer et Experiments in Art and Technology en art, les réflexions et les recherches expérimentales des avant-gardes ont souvent précédé les effets de la technologie elle-même, et préparé le terrain pour des changements culturels profonds. Riche d'anecdotes jamais gratuites, le propos parcourt aussi bien le domaine musical académique contemporain, l'expansion des techniques du son, le développement des audio-naturalistes et chasseurs de sons divers, que les interventions artistiques urbaines.

De ce panorama tout à fait convainquant, émerge la grande figure de l'artiste démiurge. En l'établissant comme repère, l'auteure en fait un principe de liaison entre des réalisations qui ne relèvent pourtant pas toujours des mêmes modalités de fonctionnement ou d'expression. Le point de vue adopte alors une perspective adossée à l'histoire de l'art. Pour l'enrichir, une suite du travail pourra intégrer davantage le cinéma qui n'est présent ici qu'à la marge ; le grand art du XXe siècle est aussi l'un des champs fructueux de l'expérimentation son-image.

7 L'espace dont nous parle Carlotta Darò est un mixage entre des matérialités, des propositions sensorielles et des pratiques. Pour les musiques instrumentales de la seconde moitié du $\mathrm{XX}^{\mathrm{e}}$ siècle, la localisation s'est affirmée comme un paramètre de composition aussi performant que les plans d'action sonore traditionnels: intensité, hauteur, timbre, harmonie et rythme. Certes, depuis longtemps les compositeurs utilisaient la spatialisation, mais ils ne disposaient que de deux modes : l'acoustique des lieux d'écoute et le placement des interprètes. Avec les techniques de reproduction et d'amplification des sons, l'espace sonore est devenu un enjeu en soi, pouvant transformer la perception même de l'espace physique le contenant. Carlotta Darò décrit les conséquences architecturales de ces avancées sonores, des cloisons invisibles aux juke-box urbains, en passant par le carrelage phonique et la ville-discothèque.

8 Le propos de l'ouvrage demeure centré sur l'artiste créateur de sons et sur les conséquences spatiales "remarquables » qui ont pu découler des expérimentations sonores. De ce fait, les relations du sonore avec l'espace sont rarement abordées à travers les autres acteurs du champ, pourtant aussi impliqués dans l'expérience, tels que les concepteurs ou les habitants. La réception n'est pas présentée comme une donnée intégrée à la production, si bien que l'ensemble des innovations et créations se 
retrouvent nivelées dans une égalité formelle sans que le moment de la perception par les auditeurs ne vienne les relativiser.

9 Certes l'ambition originelle du livre n'intègre pas une enquête de type sociologique et on comprend que la tâche descriptive des réalisations sonores - elle, parfaitement accomplie - ait mobilisé toute l'énergie. Les questions posées au cœur de l'ouvrage ouvrent sur une interrogation plus large : les notions théoriques générales auxquelles il est fait appel, comme le paysage sonore, le soundscape, l'ambiance, la ville musicale... sont-elles opératoires sans que la dimension de l'écoute, dans ses multiples modalités et ses contradictions, ne soit partie prenante de la réflexion?

Lorsque l'auteure présente les éléments de réglementation de l'acoustique des bâtiments, elle n'aborde pas le rôle des acteurs incontrôlés du sonore ou celui, plus passif, des auditeurs que constituent les habitants. Il est vrai que cela ouvre sur une autre recherche, nécessitant ses méthodes en propre. Mais l'absence du champ de la réception ordinaire, bien compréhensible ici, engendre le sentiment d'une certaine déconnexion entre l'inventivité des formes sonores élaborées et leurs retombées sur les modes de vie. Pour un effet walkman ou une diffusion de muzak, combien de tentatives (pourtant autrement jubilatoires que la musique d'ascenseur!) sont restées dans la confidentialité ?

11 On le comprend, le bémol d'impatience mentionné ici sur le caractère flou qui nimbe l'ensemble des notions théoriques convoquées sur ce champ et la difficulté de les articuler les unes par rapport aux autres, ne serait-ce que par un jeu de définitions, s'adresse moins à l'auteure qu'à notre communauté scientifique tout entière.

C'est à une consolidation de ces termes qu'appelle in fine Carlotta Darò. Avec cet ouvrage, elle apporte une pierre très constructive à ce cheminement collectif. En reliant les avancées de l'art aux processus de spatialisation, en décrivant minutieusement les progressions de la pensée contemporaine à travers les formes sonores, en rapprochant dans un même mouvement musique savante et arts des sons bruts, elle documente savamment les apports de l'esthétique à la réflexion interdisciplinaire, inévitable méthode pour progresser dans la connaissance de l'environnement sonore.

\section{AUTEURS}

\section{HENRY TORGUE}

Henry TORGUE, sociologue, diplômé de sciences politiques, docteur en études urbaines, HDR en sciences humaines - aménagement, mène de front deux activités. Chercheur au CRESSON à l'Ecole Nationale Supérieure d'Architecture de Grenoble, il travaille principalement sur la culture sonore au quotidien et sur l'imaginaire des espaces urbains contemporains. Compositeur, notamment pour la danse contemporaine et l'audiovisuel, il a enregistré 20 CDs (éditions Hopi-Mesa). Dernier ouvrage paru : Le sonore, l'imaginaire et la ville. De la fabrique artistique aux ambiances urbaines. Paris : L'Harmattan, 2012 
Mail : henry.torgue@grenoble.archi.fr

Page web : http://www.cresson.archi.fr/EQ/EQht.htm 